Sex differences in the cumulative incidence of substance use by birth cohort

Esther Colell

Albert Sánchez-Niubóa, ${ }^{a, b}$ asanchez@imim.es

Antònia Domingo-Salvany $y^{\mathrm{a}, \mathrm{b}}$ adomingo@imim.es

a Drug Abuse Epidemiology Research Group. IMIM-Hospital del Mar, Doctor Aiguader 88, E-08003

Barcelona, Spain

${ }^{\mathrm{b}}$ CIBER en Epidemiología y Salud Publica (CIBERESP), Barcelona, Spain

Corresponding Author:

Antònia Domingo-Salvany

IMIM-Hospital del Mar, Doctor Aiguader 88, E-08003 Barcelona, Spain

adomingo@imim.es Phone: +34933160700 


\section{Sex differences in the cumulative incidence of substance use by birth cohort}

\section{Keywords:}

Substance use, Cohort effect, Gender, General population survey, Spain

\section{ABSTRACT}

Background: Men present higher overall rates of substance use and abuse than women; yet, evidence suggests that an increase of substance use by the younger cohorts of women in recent decades is narrowing this gap in western societies. Moreover, younger cohorts may also be reporting earlier initiation of substance use, representing an increased risk for developing substance-related problems. With this study we intend to identify changes in the patterns of substance use of men and women in Spain for public health policy, planning and intervention.

Methods: Sex differences in the cumulative incidence of alcohol, tobacco, cannabis and cocaine were examined by birth cohort using a combined sample of individuals aged 15-64 years from eight editions of the Spanish National Survey on Drugs (1995-2009).

Results: Initiation of substance use in Spain is progressively taking place at younger ages, particularly among women. The gender-gap of life-time occurrence of substance use is narrowing (cannabis and cocaine) almost closing (alcohol) and even reversing (tobacco) in the youngest cohort.

Conclusion: These results reflect the particular evolution and trends of Spanish society regarding substance use. Women's increased use of substances and the earlier age of initiation of substance use by both sexes present particular challenges for prevention and treatment of future substance-related problems. The trends registered for legal and illegal substances would require re-evaluation of existing prevention policies.

Abstract word count: 222 


\section{INTRODUCTION}

It is well established that use of psychoactive substances in our setting is quite widespread among the general population, and that those used most prevalently are legal substances, alcohol and tobacco, followed by illegal substances such as cannabis and cocaine. In the general population men show significantly higher rates of substance use, abuse, and dependence than women, but a recent epidemiological survey worldwide registered an increase in drugs use and a narrowing of the malefemale gap in more recent cohorts, particularly in western countries (Degenhardt et al., 2008). Similarly, a literature review of birth cohort effects and gender differences in alcohol epidemiology (Keyes, Li, \& Hasin, 2011) found more problematic drinking by younger birth cohorts and a reduction of the gender gap in alcohol problems, particularly in the US and in northern and eastern Europe.

Equally, evidence suggests that the onset age of substance use has become earlier (Degenhardt, Lynskey, \& Hall, 2000; Degenhardt, Chiu, Sampson, Kessler, \& Anthony, 2007), particularly for women (Johnson \& Gerstein, 1998; Johnson \& Gerstein, 2000; Kerr, Greenfield, Bond, Ye, \& Rehm, 2007). An early onset of use of a specific substance is a risk factor for disorders related to that substance (Behrendt, Wittchen, Hofler, Lieb, \& Beesdo, 2009; Dawson, Goldstein, Chou, Ruan, \& Grant, 2008; Grucza, Norberg, Bucholz, \& Bierut, 2008; Pitkanen, Lyyra, \& Pulkkinen, 2005), although this effect has not been proved across substance classes. In contrast, a younger age at first alcohol use has been associated with increased risk of cannabis use (Behrendt et al., 2012).

According to the Spanish Observatory on Drugs 2009 report (Observatorio Español de Drogas, 2009), the use of substances in the general population is more prevalent in men with the exception of hypnotics-sedatives. Still, the differences in tobacco and alcohol use between sexes have decreased in recent years, particularly among people aged 15-34 years. Similarly, regarding illegal substances, while overall prevalence of use continues to be higher in men, differences between sexes have narrowed among the youngest, especially for cannabis.

The characteristics of substance use are determined geographically and historically and potential changes with respect to substance use and initiation arise from changes of social norms and social substance consumption behaviour at population level and of policies, laws and economic factors affecting substance availability, and related consequences (Neve, Diederiks, Knibbe, \& Drop, 1993; Brand, Saisana, Rynn, Pennoni, \& Lowenfels, 2007; Degenhardt \& Hall, 2012). Since the latter part of last century and especially in western countries, women are experiencing changes in their social role involving better opportunities for education, employment and delayed childbearing, which would have provided a social and political framework for a shift in the norms surrounding their use of substances (Bond et al., 2010) and would eventually explain an increase of substance use by women in recent cohorts (Rahav, Wilsnack, Bloomfield, Gmel, \& Kuntsche, 2006).

The objective of this paper is to present cumulative incidence rates of alcohol, tobacco, cannabis and cocaine for population groups defined by year of birth and sex in Spain. This measure, as a dynamic and therefore modifiable risk behaviour indicator, will allow us to observe birth cohort variations in men and women with respect to the age of initiation of substance use. 


\section{METHODS}

\section{Source of information}

Under the National Plan on Drugs, the Spanish health authorities have been carrying out a biennial household survey since 1995, the Encuesta Domiciliaria sobre Alcohol y Drogas en España (EDADES). EDADES is a nation-wide representative household survey of residents in Spain aged 15 to 64 years old. The present study uses data from eight editions of the survey with a combined sample size of $131,330(1995=8,888 ; 1997=12,304 ; 1999=12,234 ; 2001=14,113 ; 2003=12,033 ; 2005=27,934$;

$2007=23,715$; and $2009=20,109$ ) with $48 \%$ men and $52 \%$ women. Information on participation rates was not available for survey editions from 1995 to 2001, while for surveys from 2003 to 2009 response rates were just over 50\%. Age of first alcohol use was not asked in 1995; therefore, data on alcohol use is limited to editions 1997 to 2009.

\section{Measures}

Information on substance use was obtained through a self-administered standardised questionnaire with separate modules for each substance. For the purpose of this research we focused on the most prevalent substances: alcohol, tobacco, cannabis and cocaine, adding together the data obtained for cocaine base and cocaine powder; and excluded hypnotics-sedatives due to disparities in data collection between editions. The first question on each module was designed to identify the use of the substance ever: "have you ever tried any kind of alcoholic beverage/smoked a cigarette or any type of tobacco/used cannabis or marihuana/used cocaine base/cocaine powder?"

In each module the age of first use was registered for people reporting ever having used the substance: "How old were you when you first tried any alcoholic beverage/smoked a cigarette or any other type of tobacco/used cannabis or marihuana/used base or powder cocaine?" For analytical purposes the minimum and maximum ages of first substance use were restricted to ranges of 5 to 64 years of age for alcohol and tobacco, and of 10 to 64 for cannabis and cocaine; $99.9 \%$ of the responses fell within these ranges. An early use was defined for those initiating alcohol or tobacco use by 14 years of age, and by 16 years for cannabis, well before organic and emotional maturity is reached.

Year of birth was grouped to form three 15-year cohorts: cohort 1, born 1930-1944 $(\mathrm{N}=9,583)$; cohort 2, born 1945-1959 ( $\mathrm{N}=26,220)$; cohort 3, born 1960-1974 ( $\mathrm{N}=46,437)$; and two ten-year groups for the youngest: cohort 4, born 1975-1984 ( $N=32,498)$; and cohort 5, born 1985-1994 ( $N=16,592)$.

The cumulative occurrence is a cumulative incidence proportion estimated among surviving cohort members at the date of their survey participation (Degenhardt et al., 2007). This parameter determines the initiation of substance use and is often referred to as lifetime substance use. Since substance use in the youngest cohort is right censored as it is necessarily reported at a younger age as they have not yet reached older ages, we estimated the mean age of onset of substance use restricted to substance initiation between 10 and 24 years for each cohort in addition to the unrestricted mean age of onset (10-64), to level up all cohorts with the youngest.

\section{Statistical analysis}

Following the retrospective method developed by Gfroerer and Brodsky (1992) composite estimates of incidence proportions for each substance by sex, age of onset and birth cohort, were calculated as a weighted mean of their respective incidence figures for each survey. Weights were 
calculated as the inverse of the variance of the incidence proportion within the survey, and this variance was calculated using the non-parametric bootstrap approach with 1,000 iterations with replacement of the questionnaire respondents. The $95 \%$ confidence interval of the incidence proportion was also calculated via the non-parametric bootstrap approach with 1,000 iterations more with replacement. In all cases, survey sample design was considered. Composite incidence proportions were converted into cumulative incidence curves.

As the composite incidence proportions were distributed by age of onset of substance use, we obtained the mean age of onset considering only subjects that had started use of the specific substance. Note that the sum of incidence proportions of all ages of onset equals the life-time prevalence.

The software used for the statistical analysis was R version 2.14.0 (R Development Core Team, 2011).

\section{RESULTS}

\section{Life-time prevalence and onset of substance use}

Estimates of life-time prevalence (LTP) and onset of use of alcohol, tobacco, cannabis and cocaine by birth cohort and sex are shown in table 1 . Alcohol is the most prevalent substance for both sexes, overall LTP being $91.5 \%$ for men and $84.4 \%$ for women. The analysis by birth cohort shows that sex differences in LTP of alcohol decline in successive birth cohorts, with no significant differences between sexes from cohort 3 onwards. With respect to the onset of alcohol use, men report an earlier onset than women in each birth cohort in both restricted (10-24 years) and unrestricted (10-64 years) estimates. Major differences between the two estimates are registered for women in the two oldest cohorts, meaning that women in older cohorts started much later than men. Thus, while onset of use becomes earlier for both men and women in successive birth cohorts, the age reduction is larger among women.

LTP of tobacco use for the total sample is bigger for men (74.1\%) than for women $(58.8 \%)$, but again this outcome is nuanced when results by birth cohort are examined. Cohort 1 shows the biggest differences with men having a LTP of tobacco use over three times that of women, but the gap diminishes in subsequent birth cohorts and disappears from cohort 4. Age of onset of tobacco use declines for both sexes, each cohort reporting a lower age of onset than its predecessor, this reduction being again more manifest in women. Once more, differences between restricted and unrestricted estimates are largest for women in cohorts 1 and 2 .

For cannabis, the estimated overall LTP of men doubles that of women $(30.8 \%$ and $16.0 \%$, respectively). Analyses by birth cohort indicate a significant shift in LTP of cannabis use in cohort 3. Cohort 4 carries on this rise, especially in women, resulting in a reduction of the differences between sexes. Finally, cohort 5 shows a slight reduction of LTP in men while it continues to rise in women. However, prevalence remains significantly higher for men across birth cohorts. Estimates of age of onset of cannabis use register a decline in each successive cohort starting in cohort 3 for both sexes. Moreover, marked differences between restricted and unrestricted estimates are recorded for both men and women up to cohort 3. 
Finally, the estimated LTP of cocaine for the total sample is more than three times higher for men compared to women (7.3\% and $2.3 \%)$. As with cannabis, cohort 3 presents a major change with LTP of cocaine more than doubling that of previous cohorts. In subsequent cohorts, LTP slightly decreases for men and increases for women, but remains significantly higher for men. Interpretation of estimates of age of onset of cocaine use need to take into account the small number of observations registered in older cohorts, especially for women. Still, age of onset falls in successive cohorts from cohort 3, while substantial differences between restricted and unrestricted estimates are registered for both sexes in cohorts 1 to 3 .

\section{Cumulative incidence}

Sex-cohort variations are also clear when we examine the cumulative incidence of substance use determined by the age of initiation. Figure 1 presents the data for the four substances studied. A close examination of the results shows, in the case of alcohol, that $69 \%$ of males of cohort 1 had used alcohol by age 18 years, compared to only $26 \%$ of women. In contrast, $83 \%$ of men and $82 \%$ of women had used alcohol by the time they turned 18 in the youngest cohort. Similarly, $27 \%$ of women of the youngest cohort had used alcohol by age 14 years, compared to only 5\% in cohorts 1 and 2 . For men, the cumulative proportion for starting alcohol use by age 14 years goes from $19 \%$ in the older cohorts to $30 \%$ in the youngest. Sex-cohort variations for tobacco are even more manifest: only $6 \%$ of women had started tobacco use at age 18 years in cohort 1, whereas in cohort 5 the cumulative proportion was 55\%. In contrast, roughly the same proportion of men (50\%) had started tobacco use by the time they turned 18 in cohorts 1 and 5. Similarly, the same proportions of men and women initiated early tobacco use in the youngest cohort (23\%), but while the pattern in men has barely changed compared to older cohorts, women present a clear upward trend of early use of tobacco. With respect to cannabis, major differences are registered between older and younger cohorts as early initiation is almost nil in older cohorts for both sexes. It begins to show up in cohort 3 and becomes progressively more evident in subsequent cohorts in both sexes, especially among women. In the youngest cohort $26 \%$ of men and $19 \%$ of women had tried cannabis by the time they turned 16 . Finally, $9 \%$ of men and $4 \%$ of women had started cocaine use by age 21 in the youngest cohort, while $6 \%$ of men and only $1 \%$ of women had done so in cohort 3.

\section{DISCUSSION}

This study shows that substance use in Spain has been initiated at progressively younger ages, this trend being more intense among women. This change, while consistent across the substances examined, presents differences depending on whether the substance is legal (alcohol and tobacco) or illegal (cannabis and cocaine). Alcohol is the most prevalent substance with similar LTP for both sexes in the youngest cohort. Moreover, the gender-gap of early use of alcohol has almost closed in the youngest cohort with men and women initiating alcohol use at roughly the same age. Similarly, the growing and earlier incorporation of women to tobacco use in recent cohorts has led to an excess LTP of tobacco use among younger women. On the other hand, while estimated overall LTP of cannabis use of men doubles that of women, the gap has substantially narrowed among the youngest cohort. 
Differences between sexes in cocaine use remain considerable across cohorts from cohort 3; still, differences in the youngest cohort are smaller.

Similar studies have found comparable cohort patterns across the different substances overall in the US (Degenhardt et al., 2007) and Australia (Degenhardt et al., 2000); and by sex in the US (Johnson \& Gerstein, 1998; Johnson \& Gerstein, 2000; Kerr et al., 2007). Conversely, a recent study in Australia (Lenton, Liang, \& Chikritzhs, 2012) that controlled for the effect of right censoring, registered an overall increase in the age of onset of cannabis use in younger cohorts, pointing to a levelling off of the onset of cannabis use in that country.

Socioeconomic and cultural factors will have an effect on the prevalence of any licit or illicit substance use (Brand et al., 2007; Neve et al., 1993; Kerr, Greenfield, Bond, Ye, \& Rehm, 2004; Johnson \& Gerstein, 1998; Rahav et al., 2006) and will also determine the sequence of substance use initiation (Degenhardt et al., 2010). While changes in laws would probably affect the use of licit drugs, use of illegal drugs would be primarily influenced by drug availability, use of tobacco and alcohol at an early age, and social norms for the toleration of alcohol and other drug use (Degenhardt \& Hall, 2012).

The strong embedding of alcohol in the Spanish culture and the difficulties to establish preventive measures to limit its availability (Alcohol and Public Policy Group, 2010; Rodriguez-Martos, 2007) may account for its widespread use. Besides, the growing incorporation of the younger cohorts of women to tobacco use, along with the decline of the prevalence of tobacco among older men (Observatorio Español de Drogas, 2009) is consistent with the model of the tobacco epidemics in developed countries described by Lopez, Collishaw and Piha (1994). Briefly, this four-stage model establishes that smoking epidemics begin with a rapid increase of prevalence among males while that for women remains low. In stage two, prevalence for men reaches its peak while that for women begins to rise rapidly. Spain would currently be positioned in the third stage of the epidemic in which prevalence begins to decline in men while for women it continues to rise, before rates for both sexes decline in stage four.

In the case of illegal substances, our findings present cohort 3 as a turning point. This cohort, formed by people born between 1960 and 1974, was in their teens and twenties during the transition period and the beginning of democracy in Spain (late seventies and eighties). This period symbolised Spain's opening to new social norms that would influence the perception and availability of illegal drugs, especially cannabis. The low perception of the risks of cannabis in recent years along with the relaxation of the norms surrounding its use and its incorporation into daily life (Maldonado, 2009), has made cannabis the most prevalent illegal substance. With respect to cocaine, despite the notable increase in recent cohorts, its use is limited among the youngest, possibly due to its higher cost and the perception of it being more dangerous (Observatorio Español de Drogas, 2009).

Research on cumulative incidence of substance use and the age of initiation of such use based on cross-sectional data has several limitations. The first refers to a selective attrition process due to drug-related excess mortality. A cross-sectional sample in any given year consists of survivors to that point in time. Higher mortality among individuals in the older cohorts who initiated drug use at an early age may account for at least some of the cohort differences in cumulative incidence and in age of initiation. However, this is unlikely to explain the rather large differences in cumulative incidence found 
across birth cohorts for the different substances. In the case of cannabis, there is no evidence of significantly elevated mortality risk (Calabria, Degenhardt, Hall, \& Lynskey, 2010). Even if those who began use early had substantially higher mortality, this would be unlikely to account for cumulative incidence proportions of cannabis use by age 16 years that were $24 \%$ lower for men and $17 \%$ for females in the oldest cohort compared to the youngest (fig. 1). In the case of cocaine, major differences were observed in the three younger cohorts compared to the two older cohorts. Furthermore, while tobacco-associated mortality is apt to be especially large, this substance exhibited the smallest cohort variations in men, where the attrition process was more likely to operate. Secondly, drug use in younger birth cohorts is necessarily reported at a younger age because they have not yet reached older ages. We restricted the estimation of the mean age of onset of substance use to 10-24 years for each birth cohort thus making results comparable between cohorts as they had the same opportunity to start use. Our results registered major differences between restricted (10-24 years) and unrestricted (10-64 years) age of onset estimates in women of older cohorts for alcohol and tobacco, and in both sexes for cannabis and cocaine, congruous with onset of use of the different substances by sex across cohorts.

Also, recall bias in retrospective reporting of age of first drug use may be stronger for older cohorts as they are being asked about events that may have occurred some decades ago. Longitudinal studies of adolescents have found that estimates of the age of first use tend to increase as people grow, although the rank ordering of the different drugs persists (Labouvie, Bates, \& Pandina, 1997; Engels, Knibbe, \& Drop, 1997). In the case of tobacco, the estimated cumulative incidence use at 18 years in our study was lower for the youngest cohort than it was for its predecessor cohort. Also, distinct birth cohort trends in cumulative incidence of drug use were observed across different substance types, suggesting that the pattern of responses was not being affected by a uniform response bias.

A further limitation concerns the survey sample design based on households, as the exclusion of homeless people and residents of institutional settings might have led to a potential underreporting of early use of substances. Additionally, while response rate was similar in those survey editions for which information on participation was available, the fact that those refusing to respond shared some particular characteristics with respect to their onset of substance use cannot be ruled out, and their exclusion may have also led to an underestimation of early substance use. Disparities in data collection methods between the different survey editions have prevented the inclusion of hypnotics-sedatives in the analysis of this study. Hypnotics-sedatives are the only substance more prevalent in females and its inclusion could have enriched the interpretation of the results.

Adolescence is a critical period for initiation, experimentation, and the establishment of persistent patterns of substance use. Our findings agree with other reports on time-trend analysis in adolescent health-risk behaviours (Sweeting, Jackson, \& Haw, 2011; Mendoza, Batista Foguet, Sanchez, \& Carrasco Gonzalez, 1998; Mendoza, Lopez Perez, \& Sagrera, 2007) that document increases in risk behaviour rates and gender convergence in the 1990s. Furthermore, this study confirms the trend registered in other studies in the US and Australia that onset ages for substance use have become earlier in recent cohorts (Degenhardt et al., 2007; Degenhardt et al., 2000), and that this change has been substantially larger for women (Johnson \& Gerstein, 1998; Johnson \& Gerstein, 2000; Kerr et al., 2007). Since an early age of onset of substance use is a risk factor for substance use disorders (Behrendt 
et al., 2009; Dawson et al., 2008; Pitkanen et al., 2005), the fact that more recent birth cohorts are more likely to use both licit and illicit substances implies that more individuals from younger cohorts may be at risk of developing substance related problems. Moreover, given the steady incorporation of women to substance use in later birth cohorts, it seems that problems derived from substance abuse are no longer going to be exclusive to men. Increases of tobacco-related lung cancer mortality rates in women have been documented in countries where in men they tend to decrease or stabilise (Bray \& Weiderpass, 2010). Similarly, a recent study in Iceland (Steingrimsson, Carlsen, Sigfusson, \& Magnusson, 2012) registered a reduction of the gender gap for alcohol use disorder due to an increase in women and a decrease in men, while substance use disorders other than alcohol increased for both men and women.

Research on gender differences in the substance-related epidemiology points to significant biological and psychosocial differences that influence presentation, comorbidity, responses, progression to dependence, and treatment of substance use disorder in women (Brady \& Randall, 1999; Broome, Hurley, \& Taber, 2010; Greenfield, Back, Lawson, \& Brady, 2010; Tuchman, 2010), suggesting that women's drug use presents challenges different from those generated by men's drug use. However, it has also been noted that the use of a substance becoming more widespread and normative complicates the analysis of its effects (Kessler, 2004; Keyes, Martins, Blanco, \& Hasin, 2010; Weiss, Mirin, Griffin, \& Michael, 1988). Consequently, changes in the prevalence and social meaning of women's substance use would require further research to reassess their differential vulnerability.

In conclusion, our results reflect the particular evolution and main trends of substance use in Spain. The overall increase and earlier onset of substance use and the change in women's substance use patterns has important implications for prevention and treatment. Policies and preventive measures regarding the use and availability of legal and illegal substances need to be reassessed, particularly those addressed to young people. 


\section{Funding:}

This work was supported by the Delegación del Gobierno para el Plan Nacional sobre Drogas of the Ministerio de Salud y Política Social, grant numbers: PNSD 2007/I044 and 2011/1073; FIS-Redes de investigación cooperativa, grant number: RD06/0001/1018; and AGAUR, grant number: 2009 SGR 00718. Albert Sánchez-Niubó was supported by Instituto de Salud Carlos III, grant number: CA08/00214.

\section{Acknowledgements:}

The work was partially developed within the framework of the EU project JUST/2010/DPIP/AG/1410: New methodological tools for policy and programme evaluation.

We are grateful to Dave MacFarlane for his help with the English language usage in this paper. 


\section{References}

Alcohol and Public Policy Group (2010). Alcohol: no ordinary commodity--a summary of the second edition. Addiction, 105, 769-779.

Behrendt, S., Beesdo-Baum, K., Hofler, M., Perkonigg, A., Buhringer, G., Lieb, R. et al. (2012). The relevance of age at first alcohol and nicotine use for initiation of cannabis use and progression to cannabis use disorders. Drug and Alcohol Dependence, 123, 48-56.

Behrendt, S., Wittchen, H. U., Hofler, M., Lieb, R., \& Beesdo, K. (2009). Transitions from first substance use to substance use disorders in adolescence: is early onset associated with a rapid escalation? Drug and Alcohol Dependence, 99, 68-78.

Bond, J. C., Roberts, S. C., Greenfield, T. K., Korcha, R., Ye, Y., \& Nayak, M. B. (2010). Gender differences in public and private drinking contexts: a multi-level GENACIS analysis. International Journal of Environmental Research and Public Health, 7, 2136-2160.

Brady, K. T. \& Randall, C. L. (1999). Gender differences in substance use disorders. Psychiatric Clinics of North America, 22, 241-252.

Brand, D. A., Saisana, M., Rynn, L. A., Pennoni, F., \& Lowenfels, A. B. (2007). Comparative analysis of alcohol control policies in 30 countries. PLoS.Med., 4, e151.

Bray, F. I. \& Weiderpass, E. (2010). Lung cancer mortality trends in 36 European countries: secular trends and birth cohort patterns by sex and region 1970-2007. International Journal of Cancer, 126, 1454-1466.

Broome, M. V., Hurley, R. A., \& Taber, K. H. (2010). Substance use disorders: do males and females have differential vulnerability? Journal of Neuropsychiatry and Clinical Neurosciences, 22, iv.

Calabria, B., Degenhardt, L., Hall, W., \& Lynskey, M. (2010). Does cannabis use increase the risk of death? Systematic review of epidemiological evidence on adverse effects of cannabis use. Drug and Alcohol Review, 29, 318-330.

Dawson, D. A., Goldstein, R. B., Chou, S. P., Ruan, W. J., \& Grant, B. F. (2008). Age at first drink and the first incidence of adult-onset DSM-IV alcohol use disorders. Alcoholism: Clinical and Expimental Research, 32, 2149-2160.

Degenhardt, L., Chiu, W. T., Sampson, N., Kessler, R. C., \& Anthony, J. C. (2007). Epidemiological patterns of extra-medical drug use in the United States: Evidence from the National Comorbidity Survey Replication, 2001-2003. Drug and Alcohol Dependence, 90, 210-223.

Degenhardt, L., Chiu, W. T., Sampson, N., Kessler, R. C., Anthony, J. C., Angermeyer, M. et al. (2008). Toward a global view of alcohol, tobacco, cannabis, and cocaine use: findings from the WHO World Mental Health Surveys. PLoS.Med., 5, e141.

Degenhardt, L., Dierker, L., Chiu, W. T., Medina-Mora, M. E., Neumark, Y., Sampson, N. et al. (2010). Evaluating the drug use "gateway" theory using cross-national data: consistency and associations of the order of initiation of drug use among participants in the WHO World Mental Health Surveys. Drug and Alcohol Dependence, 108, 84-97.

Degenhardt, L. \& Hall, W. (2012). Extent of illicit drug use and dependence, and their contribution to the global burden of disease. The Lancet, 379, 55-70. 
Degenhardt, L., Lynskey, M., \& Hall, W. (2000). Cohort trends in the age of initiation of drug use in Australia. Aust.N.Z.J.Public Health, 24, 421-426.

Engels, R. C., Knibbe, R. A., \& Drop, M. J. (1997). Inconsistencies in adolescents' self-reports of initiation of alcohol and tobacco use. Addict.Behav., 22, 613-623.

Gfroerer, J. \& Brodsky, M. (1992). The incidence of illicit drug use in the United States, 19621989. British Journal of Addiction, 87, 1345-1351.

Greenfield, S. F., Back, S. E., Lawson, K., \& Brady, K. T. (2010). Substance abuse in women. Psychiatric Clinics of North America, 33, 339-355.

Grucza, R. A., Norberg, K., Bucholz, K. K., \& Bierut, L. J. (2008). Correspondence between secular changes in alcohol dependence and age of drinking onset among women in the United States. Alcohol Clin.Exp.Res., 32, 1493-1501.

Johnson, R. A. \& Gerstein, D. R. (1998). Initiation of use of alcohol, cigarettes, marijuana, cocaine, and other substances in US birth cohorts since 1919. American Journal of Public Health, 88, 2733.

Johnson, R. A. \& Gerstein, D. R. (2000). Age, period, and cohort effects in marijuana and alcohol incidence: United States females and males, 1961-1990. !Lost Data, 35, 925-948.

Kerr, W. C., Greenfield, T. K., Bond, J., Ye, Y., \& Rehm, J. (2004). Age, period and cohort influences on beer, wine and spirits consumption trends in the US National Alcohol Surveys. Addiction, 99, 1111-1120.

Kerr, W. C., Greenfield, T. K., Bond, J., Ye, Y., \& Rehm, J. (2007). Age-period-cohort influences on trends in past year marijuana use in the US from the 1984, 1990, 1995 and 2000 National Alcohol Surveys. Drug and Alcohol Dependence, 86, 132-138.

Kessler, R. C. (2004). The epidemiology of dual diagnosis. Biol.Psychiatry, 56, 730-737.

Keyes, K. M., Li, G., \& Hasin, D. S. (2011). Birth cohort effects and gender differences in alcohol epidemiology: a review and synthesis. Alcohol Clin.Exp.Res., 35, 2101-2112.

Keyes, K. M., Martins, S. S., Blanco, C., \& Hasin, D. S. (2010). Telescoping and gender differences in alcohol dependence: new evidence from two national surveys. Am.J.Psychiatry, 167, 969-976.

Labouvie, E., Bates, M. E., \& Pandina, R. J. (1997). Age of first use: its reliability and predictive utility. J.Stud.Alcohol, 58, 638-643.

Lenton, S., Liang, W., \& Chikritzhs, T. (2012). Evidence of increasing age of onset of cannabis use among younger Australians. Addiction., 107, 650-657.

Lopez, A. D., Collishaw, N. E., \& Piha, T. (1994). A descriptive model of the cigarette epidemic in developed countries. Tob Control, 242-247.

Maldonado, R. (2009). [Cannabis. Risk/benefit]. Medicina Clínica (Barcelona), 132, 625-626. 
Mendoza, B. R., Batista-Foguet, J. M., Sanchez, G. M., \& Carrasco-Gonzalez, A. M. (1998). [The consumption of tobacco, alcohol and other drugs by adolescent Spanish students]. Gaceta Sanitaria, 12, 263-271.

Mendoza, R., Lopez-Perez, P., \& Sagrera, M. R. (2007). [Gender differences in the evolution of adolescent's tobacco consumption in Spain (1986-2002)]. Adicciones, 19, 273-287.

Neve, R. J., Diederiks, J. P., Knibbe, R. A., \& Drop, M. J. (1993). Developments in drinking behavior in The Netherlands from 1958 to 1989, a cohort analysis. Addiction, 88, 611-621.

Observatorio Español de Drogas (2009). Informe 2009. Situación y tendencias de los problemas de drogas en España. Madrid. Ministerio de Sanidad y Política Social.

Pitkanen, T., Lyyra, A. L., \& Pulkkinen, L. (2005). Age of onset of drinking and the use of alcohol in adulthood: a follow-up study from age 8-42 for females and males. Addiction, 100, 652-661.

R Development Core Team (2011). R: A Language and Environment for Statistical Computing (Version 2.10.0) [Computer software]. Vienna, Austria: R Foundation for Statistical Computing.

Rahav, G., Wilsnack, R., Bloomfield, K., Gmel, G., \& Kuntsche, S. (2006). The influence of societal level factors on men's and women's alcohol consumption and alcohol problems. Alcohol Alcohol Suppl, 41, i47-i55.

Rodriguez-Martos, A. (2007). [Why is it so difficult to legislate on alcohol in Spain?]. Adicciones, $19,325-331$.

Steingrimsson, S., Carlsen, H. K., Sigfusson, S., \& Magnusson, A. (2012). The changing gender gap in substance use disorder: A total population based study of psychiatric inpatients. Addiction. doi: 10.1111/j.1360-0443.2012.03954.x.

Sweeting, H., Jackson, C., \& Haw, S. (2011). Changes in the socio-demographic patterning of late adolescent health risk behaviours during the 1990s: analysis of two West of Scotland cohort studies. BMC.Public Health, 11, 829.

Tuchman, E. (2010). Women and addiction: the importance of gender issues in substance abuse research. J.Addict.Dis., 29, 127-138.

Weiss, R. D., Mirin, S. M., Griffin, M. L., \& Michael, J. L. (1988). Psychopathology in cocaine abusers. Changing trends. J.Nerv.Ment.Dis., 176, 719-725. 
Figure 1. Cumulative incidence proportions of alcohol, tobacco, cannabis and cocaine by sex and birth cohort.*
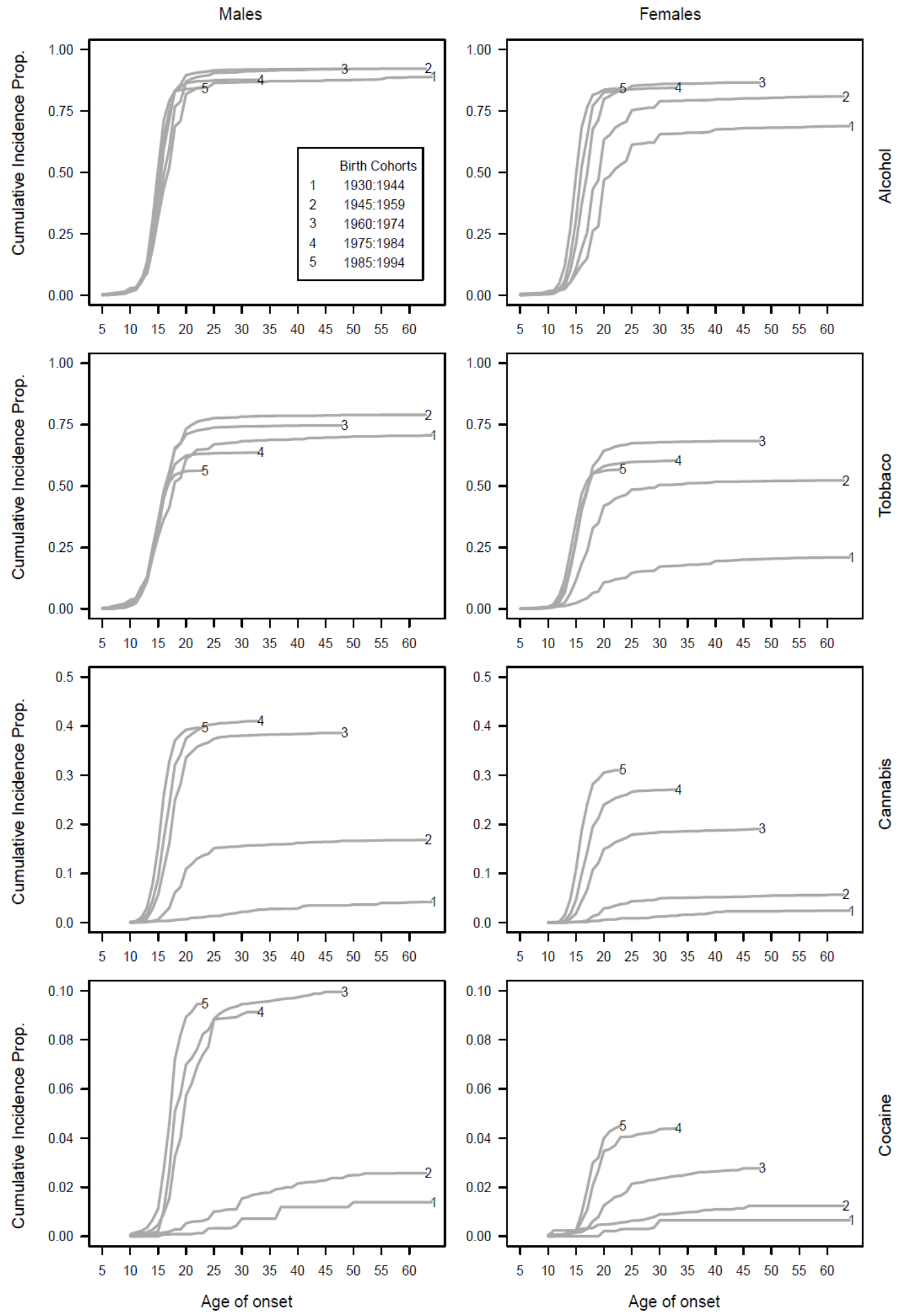

*Graph scales differ by substance according to the highest cumulative incidence. 
Table 1. Estimated life-time prevalence (LTP) and mean age of onset of alcohol, tobacco, cannabis and cocaine by birth cohort and sex ${ }^{a}$.



a $\mathrm{N}$ are from raw data, \%and means from weighted data.

${ }^{\mathrm{b}} \mathrm{Mean}$ age of onset of substance use 10-64 years

c Mean age of onset of substance use restricted to $10-24$ years

${ }^{\mathrm{a}} \mathrm{Cl}$ : Confidence Interval 